\title{
Los gastos públicos de promoción de los destinos y la demanda turística interior en España*
}

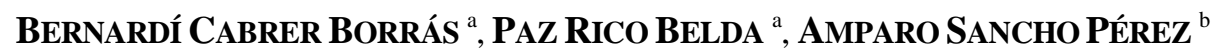 \\ a Universidad de Valencia, Facultad CC.EE., Avda. de los naranjos, s/n, 46022 Valencia, España. E- \\ mail: cabrer@uv.es, paz.rico@uv.es \\ ${ }^{b}$ Universidad de Valencia, Instituto de Economía Internacional, Avda. de los naranjos, s/n, 46022 \\ Valencia, España.E-mail: amparo.sancho@uv.es
}

\begin{abstract}
RESUMEN
A pesar del elevado peso de la demanda interna en el sector turístico español, son escasos los trabajos que se centran en el análisis de los flujos turísticos internos. Asimismo, tampoco es abundante la literatura que aborda el impacto que los gastos públicos de promoción del destino tienen sobre la demanda turística. Este artículo pretende abordar estos dos aspectos y, en concreto, se estudian los factores determinantes de la demanda turística del mercado interior en España, utilizando un modelo gravitatorio, poniendo especial atención en los gastos públicos de promoción de los destinos turísticos. Para ello se ha utilizado un panel de datos configurado por 4046 observaciones, procedentes de las matrices origen y destino de viajeros entre las diecisiete comunidades autónomas a lo largo del periodo 2000-2013. Los resultados obtenidos permiten concluir que el gasto promocional de los destinos turísticos es una variable relevante en la explicación de los flujos turísticos internos. Asimismo, se pone de relieve que los gastos promocionales tienen mayor efecto sobre los turistas locales que sobre los procedentes de otras áreas turísticas.
\end{abstract}

Palabras clave: Turismo interior, gasto promocional, modelo gravitatorio, efecto frontera, datos de panel.

\section{Public Promotional Spending and Domestic Tourism Demand in Spain}

\begin{abstract}
Despite the high proportion of domestic demand in the Spanish tourism sector, few studies focus on analysing domestic tourism flows. Nor there is much research examining the impact of public promotional spending on tourism demand. This article aims to address these two aspects by examining the determinants of tourism demand from the domestic Spanish market, using a gravity model and with particular emphasis on public promotional spending. To this end, we used a panel data set of 4046 observations from origin and destination matrices of travellers between the seventeen Spanish regions over the period 2000-2013. The results indicate that promotional spending is a relevant variable in the explication of domestic tourism flows, and also highlight that promotional spending has a greater effect on local tourists than on those from other tourist regions.
\end{abstract}

Keywords: Domestic Tourism, Promotional Spending, Gravity Model, Border Effect, Panel Data.

\section{Clasificación JEL: C23, R12, L83}

\footnotetext{
${ }^{*}$ Los autores están muy agradecidos a las ayudas dadas por los evaluadores anónimos que han proporcionado nuevas ideas y comentarios que han mejorado sensiblemente esta aportación y al Ministerio de Economía y Competitividad: CSO2013-43054-R.
}

Artículo recibido en mayo de 2016 y aceptado en julio de 2016

Artículo disponible en versión electrónica en la página www.revista-eea.net, ref. ə-34305 


\section{INTRODUCCIÓN}

Los modelos de demanda turística se han centrado en el análisis de las variables relevantes que explican la evolución de la actividad turística, con el fin de ajustar el negocio turístico a las necesidades de la demanda esperada. Estos modelos tienen por objetivo, entre otros, explicar las causas de las variaciones en la demanda turística, evaluar los efectos de las políticas públicas y predecir la evolución futura de la demanda. Generalmente, los modelos de predicción de demanda turística relacionan esta variable con el nivel de la renta de las áreas emisoras y con la relación real de los precios entre las regiones emisoras y las receptoras (Crouch, 1995; Witt y Witt, 1995; Lim, 2006 y Song y Li, 2008) ${ }^{1}$.

Como indican Juaneda y Riera (2011), la literatura ha puesto de manifiesto la existencia de dos grandes grupos de métodos cuantitativos orientados a determinar y predecir la demanda turística: los modelos causales y los modelos de series temporales univariantes. Con respecto a los modelos de series temporales univariantes, como indican Song y Li (2008), éstos fueron inicialmente muy utilizados para predecir la demanda turística a corto plazo, con una clara utilización de la metodología propuesta por Box y Jenkins (1970). Entre otros trabajos, cabe citar Almagro, 1979; Otero, 1996; Akal, 2004; Wong et al., 2007; Chu, 2008; Lee et al., 2008 y Coshall, 2009. Posteriormente, los investigadores intentaron mejorar las predicciones que proporcionaban los modelos ARIMA introduciendo extensiones. De esta forma, Goh y Law (2002) utilizaron modelos SARIMA multivariantes, que incluían funciones de intervención para captar los efectos spillover en la demanda turística. Chan et al. (2005) aplicaron modelos GARCH y Preez y Witt (2003) consideraron el análisis de intervención en las series temporales. Asimismo, también cabe destacar, entre otras aplicaciones, el análisis de los impactos derivados de eventos extraordinarios, como fueron los atentados del 11 de septiembre de 2001 y su incidencia en la demanda turística (Cabrer e Iranzo, 2007).

En la modelización de la actividad turística, también surgieron los modelos gravitatorios, para analizar el comportamiento de los mercados turísticos, a partir de la intensidad y dirección de los flujos turísticos entre regiones o países. Los modelos gravitatorios explican la demanda turística entre dos destinos como una atracción entre dos zonas o regiones, siendo la variable distancia la única referencia (Malamud, 1973). Siguiendo a Witt y Witt (1995), los modelos gravitatorios se utilizaron ampliamente en los años sesenta y setenta. Inicialmente estos trabajos modelizaban la demanda a partir de dos variables: la atracción entre las masas críticas de población y la variable fricción o distancia entre el punto de origen y el de destino. La limitación de estos modelos es evidente, ya que la atracción entre

\footnotetext{
${ }^{1}$ En estos artículos se puede encontrar una revisión exhaustiva de la literatura sobre la modelización y predicción de la demanda turística.
} 
dos zonas o regiones no es indicativo de atracción turística, puesto que ésta depende de otros factores tan importantes como son los económicos, la calidad del destino, las preferencias de los turistas, las comunicaciones e infraestructuras, entre otros. Quizá la falta de un marco teórico explique que estos modelos dejasen de utilizarse en los años ochenta y noventa ${ }^{2}$.

Recientemente, como indican Morley et al. (2014), los modelos gravitatorios han experimentado un resurgimiento y han aparecido trabajos que introducen nuevas variables y factores determinantes de los flujos turísticos, tales como son las diferencias culturales entre el origen y el destino, la calidad de las infraestructuras de transporte y el tipo de turismo. Así, Imm Ng et al. (2007) evalúan el efecto que tienen las diferencias culturales sobre la decisión de gastar en turismo y sobre la elección de destino. En esta misma línea, Khadaroo y Seetanah (2008) analizan el impacto que las infraestructuras de transporte tienen sobre el crecimiento del turismo internacional y la intensidad de los flujos bilaterales de turismo. Por su parte, Rosselló y Santana-Gallego (2014) estudian la importancia que tiene el cambio climático en la demanda de turismo internacional. Un elemento común a todos estos trabajos es que se centran en los flujos turísticos internacionales, sin atender a los movimientos interiores. De hecho, resulta muy difícil encontrar en la literatura estudios que analicen los flujos generados por el turismo interior y menos aún en consideración con el flujo intra-regional. Los escasos trabajos encontrados en el análisis del turismo doméstico se han realizado en el contexto de modelos input-output (Usach, 1998 y Eriksen y Ahmt, 1999) o bien con series temporales (Athanasopoulos y Hyndman, 2008), pero no con modelos econométricos con datos de corte transversal.

En el caso de España existen abundantes trabajos que analizan la demanda turística internacional, entre los que cabe mencionar las aportaciones de Alegre y Pou, 2004; Cañada, 2007; Aznar y Nicolini, 2007 y Garín-Muñoz, 2008, pero se le ha prestado menor atención a los flujos turísticos internos, a pesar de la importancia que tienen. En este sentido, Hatanaka (2015) indica que en el año 2012 tan solo el 7,7\% del total de viajes realizados por residentes en España fueron al extranjero. Entre los trabajos que han analizado los flujos turísticos internos en España cabe citar los de Largo, 1976; Cañada, 2002; Millán, 2004; De la Mata y Llano, 2010 y 2012, Guardia et. al., 2014 y Priego et al., 2015. Ahora bien, ninguno de estos trabajos considera los gastos públicos de promoción turística, como variable relevante en la determinación de la demanda turística interna de una región. Dado que la distribución de los turistas dentro del territorio español es muy heterogénea, y tan sólo cuatro comunidades autónomas, Andalucía, Cataluña, la Comunidad Valenciana y Madrid, aglutinan más del 50\% de la demanda interna

\footnotetext{
${ }^{2}$ En este sentido, los artículos de Anderson y Van Wincoop (2003), Anderson (2011) y Bertoli y Fernández-Huertas (2013) cuestionan la consistencia de los estimadores si no se corrigen los problemas de omisión de variables relevantes, que caracterizan a los modelos gravitatorios.
} 
de España, hace pensar que existe algún factor adicional implícito en la demanda, como pueden ser los gastos de promoción, que puede influir sobre la distribución de los turistas a escala nacional. En el presente trabajo se ha considerado el gasto público en promoción turística, de los gobiernos locales y autonómicos, como un factor catalizador de la demanda. Hay que tener en cuenta que la promoción turística abarca una gran variedad de objetivos, entre los que destacan: fomentar el atractivo turístico de un destino, dar a conocer la oferta turística existente, captar eventos de cierta relevancia para el destino e incentivar la implicación de todos los profesionales relacionados con la actividad turística, para impulsar y atraer un mayor número de visitantes.

El objetivo de este artículo es, por todo lo anteriormente expuesto, comprobar la capacidad que poseen los gastos públicos de promoción de un destino turístico en el fomento de la demanda turística. A tal fin se va a utilizar un modelo gravitatorio donde se incorpora, además de los gastos de promoción turística, factores que tradicionalmente se han utilizado en otros trabajos y que son necesarios para ejercer una cierta atracción de un destino turístico.

La estructura del este trabajo es la siguiente. Tras esta introducción, en el segundo apartado se presenta el modelo de demanda turística empleado y los datos utilizados. En el tercer apartado se analizan los resultados del modelo de gravedad propuesto y estimado. Finalmente, en el último apartado se recogen las principales conclusiones del trabajo.

\section{METODOLOGÍA}

\subsection{Modelo}

El fundamento de los modelos gravitatorios tradicionales ${ }^{3}$ se basa en una adaptación de la ley de la gravitación de Newton, relativa a la fuerza de atracción entre dos objetos en función de sus masas y de la distancia que los separa ${ }^{4}$. Se puede considerar que la atracción entre dos zonas o regiones, $i$ y $j$, que denotamos por $\left(F_{i j}\right)$, es directamente proporcional a las masas $P_{i}$ y $P_{j}$ que las componen e inversamente proporcional a la distancia entre ellas $\left(D_{i j}\right)$ :

$$
F_{i j}=k \frac{P_{i}^{\alpha} P_{j}^{\beta}}{D_{i j}^{\delta}}
$$

\footnotetext{
${ }^{3}$ El modelo gravitatorio también se puede justificar a partir de la Teoría de la Utilidad del Consumidor, ver Morley et al.(2014). En este trabajo se ha optado por el enfoque tradicional, dadas las características de la información estadística utilizada.

4 En concreto, la fuerza de atracción entre dos objetos es directamente proporcional al producto de sus masas e inversamente proporcional al cuadrado de la distancia entre ellos.
} 
Donde $k, \alpha, \beta$ y $\gamma$ son constantes ${ }^{5}$.

Al considerar la atracción turística entre dos zonas, la variable atracción recoge el número de viajes que tienen lugar entre la zona $i$, o de destino, y la zona $j$, o de origen (Witt y Witt, 1995), la variable $P_{i}$ es la población de la zona de destino, mientras que $P_{j}$ es la población de la zona de origen y $D_{i j}$ la distancia entre las dos zonas.

Al tomar logaritmos y añadiendo un término de perturbación, el modelo gravitatorio básico se recoge a través de la ecuación:

$$
\ln F_{i j}=\beta_{1}+\beta_{2} \ln P_{i}+\beta_{3} \ln P_{j}+\beta_{4} \ln D_{i j}+u_{i j}
$$

Donde $\beta_{1}=\ln k, \beta_{2}=\alpha, \beta_{3}=\beta, \beta_{4}=-\delta$ y $u_{i j}$ es una perturbación aleatoria normalmente distribuida.

La versión aumentada del modelo gravitatorio quedaría recogida por la siguiente ecuación de comportamiento:

$$
\begin{aligned}
& \ln F_{i j t}=\beta_{1}+\sum_{m=1}^{M} \beta_{m} \ln F D_{i t}+\sum_{n=1}^{N} \beta_{n} \ln F O_{j t}+\sum_{p=1}^{P} \beta_{p} \ln F F_{i j t} \\
& +\sum_{i=1}^{17} \beta_{i} D_{i}+\sum_{j=1}^{17} \beta_{j} D_{j}+\sum_{t=1}^{14} \beta_{t} D_{t}+u_{i j t}
\end{aligned}
$$

Donde $F_{i j t}$ recoge el efecto de atracción del destino $i$ sobre la zona origen $j$ en el momento t, $F D_{i t}$ son los factores de atracción de la zona de destino $i$ en t, $F O_{j t}$ son los factores de atracción procedentes de la zona de origen $j$ en $\mathrm{t}$ y $F F_{i j t}$ son los factores de fricción o costes del desplazamiento entre las dos zonas en el momento t. $D_{i}$ y $D_{j}$ son variables dummies referentes a las CCAA de destino y de origen, respectivamente, y $D_{t}$ son variables dummies temporales.

Las variables dummies referentes a las CCAA de origen y de destino y las temporales se incluyen con el fin de recoger lo que la literatura ha denominado multilateral resistance term (Anderson y Van Wincoop, 2003, 2004; Bertoli et al., 2013), cuya omisión puede generar problemas de consistencia en la estimación del modelo.

Además, se introduce la dinámica en el modelo propuesto con el fin de captar el efecto fidelización del destino. Para ello se incluye como regresor la variable endógena desfasada, tal y como proponen Fuji y Mak, 1981; Martin y

\footnotetext{
${ }^{5}$ La ley de Newton considera que $\alpha$ y $\beta$ son iguales a 1 y $\delta$ es igual a 2 .
} 
Witt, 1987; Garín-Muñoz, 2011 y Massida y Etzo, 2012, fundamentada por la persistencia en los hábitos de los turistas o por las preferencias interdependientes. La menor incertidumbre asociada con las vacaciones en un destino conocido y la fidelización al destino justificarían la aparición de estos hábitos. Por lo que respecta a las preferencias interdependientes, hay que tener en cuenta que a la hora de viajar se suele considerar, como factor determinante, las experiencias de personas de nuestro entorno y se elige destinos sobre los que se tiene alguna referencia y/o conocimiento.

Asimismo, las rigideces por el lado de la oferta, como consecuencia de que los cambios en las infraestructuras turísticas de los destinos requieren tiempo y de que el mecanismo de ajuste no es automático, justifican la presencia de la variable endógena desfasada en la función de la demanda turística. Por su parte, Morley (1998) considera que muchos estudios empíricos sobre demanda turística presentan el problema de no considerar su estructura dinámica. Asimismo, el modelo dinámico permite calcular elasticidades a corto y largo plazo.

Adicionalmente, siguiendo la literatura de "efecto frontera" (Anderson y Van Wincoop, 2003; McCallum, 1995; Okubo, 2004; Gil-Pareja et al., 2005 y Requena y Llano, 2010), el modelo propuesto incluye una variable dummy, que toma valor uno cuando la región de origen y la destino es la misma y cero en caso contrario, denominada $S_{i j}$. Esta variable permitirá cuantificar el efecto frontera, que mide el atractivo que tiene la propia región para el turista local. Asimismo, se ha incluido una variable dummy que recoge la contigüidad entre las CCAA, de tal forma que la variable Cont $_{i j}$ toma valor uno cuando dos CCAA son contiguas y cero en el resto de casos.

En la especificación final también se han introducido variables binarias que recogen la condición de capitalidad del Estado, de insularidad y de tener costa, distinguiendo entre costa mediterránea frente no costa mediterránea, y costa atlántica frente no atlántica. De la Mata y Llano (2010) introducen dos variables dummies relacionadas con la insularidad, con el objetivo de captar las especificidades que pudieran existir en los flujos monetarios tanto del origen como del destino en las islas ${ }^{6}$. Este mismo argumento puede servir para el resto de dummies.

En la literatura empírica, frecuentemente se ha utilizado como variable en-

\footnotetext{
${ }^{6}$ De la Mata y Llano (2010) argumentan que el signo esperado del coeficiente de la variable dummy cuando las islas son el destino de los flujos turísticos es positivo, dado el atractivo que pueden presentar y que no vendría captado por las variables de control. Por su parte, el signo del coeficiente de la variable dummy cuando las islas son el origen está menos claro. Por un lado, las necesidades de turismo costero estarían satisfechas, lo que implicaría un signo negativo pero, por otro lado, las subvenciones de los viajes a la península pueden incentivar los flujos turísticos hacia otras CCAA y, por tanto, esperar un signo positivo del coeficiente.
} 
dógena el número de viajeros, los días de estancia y el gasto turístico (Sheldon y Var, 1985; Kim y Fesenmaier, 1990; Zhan y Jensen, 2007; Nicolau, 2008 y De la Mata y Llano, 2010). Con respecto a las variables explicativas, las más utilizadas han sido el nivel de renta, los precios relativos del turismo, los costes de transporte y factores cualitativos de la región de destino, tales como el clima. Además, otros trabajos empíricos han considerado como variables explicativas de la demanda de turismo los gastos de promoción (Uysal y Crompton, 1984, Witt y Martin, 1987, Sheldon y Var, 1985, Crouch et al., 1992 y Lim, 1997). En este sentido, Patuelli et al. (2014) incluyen, como factor de la demanda de turismo, el gasto en actividades de recreo y/o culturales, como un indicador de la inversión de las autoridades locales para atraer a los turistas.

En este trabajo, el modelo de gravedad especificado incluye como variable endógena el número de viajeros registrados en la comunidad de destino $i$ procedente de la zona de origen $j$ a lo largo del periodo muestral $\left(F_{i j t}\right)$, mientras que las variables exógenas consideradas son el factor de inercia $\left(F_{i j t-1}\right)$, el PIB per cápita a precios constantes de la comunidad autónoma de origen $\left(P I B_{j t}\right)$, el PIB per cápita a precios constantes de la comunidad autónoma de destino $\left(P I B_{i t}\right)^{7}$, el gasto de promoción turística de la comunidad autónoma de destino $\left(G P_{i t}\right)$, el nivel de precios hoteleros en la comunidad autónoma de destino ${ }^{8}\left(P H_{i t}\right)$, el número de plazas hoteleras de la comunidad autónoma de destino $\left(N P_{i t}\right)$, la distancia en kilómetros entre la zona de origen y la de destino ( $\left.D_{i j}\right)$, las variables dummies que recogen el efecto frontera $\left(S_{i j}\right)$, la contigüidad ( Cont $\left._{i j}\right)$, la insularidad ( $I s l a_{j}$ y $\left.I s l a_{i}\right)$, la capitalidad del Estado ( $\operatorname{Mad}_{j}$ y $\left.\operatorname{Mad}_{i}\right)$, tener costa mediterránea $\left(C M_{j}\right.$ y $\left.C M_{i}\right)$ y tener costa atlántica $\left(C A_{j}\right.$ y $\left.C A_{i}\right)$.

Toda la información de las variables han sido obtenidas del INE, a excepción de los gastos de promoción de los destinos turísticos, que proceden de la Intervención General de la Administración del Estado, y la distancia entre las CCAA, que se ha obtenido de la página web distance.to.

De esta forma, el modelo finalmente especificado, con la inclusión de la variable inercia, quedaría definido por la ecuación:

\footnotetext{
${ }^{7}$ El PIB per cápita de la zona de destino puede considerarse como una proxy del nivel de desarrollo de la CCAA y, por tanto, se ha incluido en el modelo, tal y como hacen Guardia et al. (2014).

${ }^{8}$ Cabe indicar que los precios hoteleros presentan una evolución distinta para cada CCAA a lo largo del periodo analizado.
} 


$$
\begin{aligned}
& \ln F_{i j t}=\beta_{1}+\beta_{2} \ln F_{i j t-1}+\beta_{3} \ln P I B_{j t}+\beta_{4} \ln P I B_{i t}+\beta_{5} \ln G P_{i t}+\beta_{6} \ln P H_{i t} \\
& +\beta_{7} \ln N P_{i t}+\beta_{8} \ln D_{i j}+\beta_{9} S_{i j}+\beta_{10} \text { Cont }_{i j}+\beta_{11} \text { Isla }_{j}+\beta_{12} \text { Isla }_{i}+\beta_{13} \text { Mad }_{j}+\beta_{14} \text { Mad }_{i} \\
& +\beta_{15} C M_{j}+\beta_{16} C M_{i}+\beta_{17} C A_{j}+\beta_{18} C A_{i}+u_{i j t}
\end{aligned}
$$

En el estudio realizado se dispone de catorce matrices origen y destino, entre las diecisiete comunidades autónomas (CCAA) de España, correspondientes al periodo 2000-2013, lo cual configura un panel de una muestra de 4046 observaciones.

Finalmente, con el objetivo de analizar cómo interactúan los gastos públicos de promoción con las variables que recogen las distintas zonas de destino del turismo interior, se va analizar la interacción de éstos con la distancia kilométrica, entre la comunidad de origen y la de destino, y con la procedencia de los turistas, a saber, si proceden de una comunidad contigua o si proceden de la misma comunidad.

\subsection{Análisis descriptivo de los datos}

En la Tabla 1 se recoge el volumen medio de viajeros de cada comunidad autónoma, para el periodo muestral considerado. La segunda columna de esta tabla presenta el porcentaje de los viajeros de las CCAA con destino a la misma comunidad. Se aprecia que la mayor endogamia se produce en Andalucía, donde el porcentaje de turistas que eligen la misma comunidad es aproximadamente del 60\%, seguida de Canarias, por razones de insularidad, Galicia, Cataluña y la Comunidad Valenciana. Por su parte, las CCAA más exogámicas son Navarra, La Rioja, Cantabria y Madrid.

\begin{tabular}{|c|c|c|c|c|c|}
\hline & Misma Región & $\%$ sobre viajeros & Destino & Origen & Saldo \\
\hline Andalucía & 4155504 & 59.75 & 3996549 & 2798914 & 1197635 \\
\hline Aragón & 297673 & 25.71 & 1402952 & 859963 & 542989 \\
\hline Asturias & 170095 & 15.89 & 996634 & 900172 & 96461 \\
\hline Baleares & 213252 & 30.64 & 1080901 & 482789 & 598111 \\
\hline Canarias & 567733 & 51.73 & 1393467 & 529719 & 863748 \\
\hline Cantabria & 65351 & 10.76 & 787967 & 542005 & 245962 \\
\hline Castilla y León & 571028 & 27.29 & 2686966 & 1521686 & 1165280 \\
\hline Castilla - La Mancha & 255973 & 17.95 & 1354472 & 1169767 & 184704 \\
\hline Cataluña & 3054867 & 44.62 & 2626247 & 3792194 & -1165948 \\
\hline C. Valenciana & 1322705 & 35.05 & 2983467 & 2450573 & 532895 \\
\hline Extremadura & 133314 & 17.31 & 824306 & 636904 & 187402 \\
\hline Galicia & 992521 & 44.61 & 1651126 & 1232414 & 418712 \\
\hline Madrid & 920113 & 10.73 & 3622942 & 7652966 & -4030024 \\
\hline Murcia & 152160 & 16.12 & 664170 & 791723 & -127553 \\
\hline Navarra & 50845 & 8.95 & 483536 & 517536 & -34001 \\
\hline País Vasco & 311496 & 14.52 & 1011741 & 1833328 & -821587 \\
\hline La Rioja & 14668 & 4.57 & 389229 & 306637 & 82593 \\
\hline
\end{tabular}

Tabla 1

Valores medios de viajeros por comunidad autónoma del periodo 2000-2013

Fuente: Elaboración propia a partir de los datos del INE. 
La Tabla 1 también recoge el saldo o flujo neto resultante entre los viajeros de destino y los de origen de cada una de las CCAA. Como se puede observar, las CCAA cuyo saldo es negativo, y por tanto son emisoras de turistas, son Madrid, el País Vasco, Cataluña, Murcia y Navarra. Estas CCAA emisoras netas de viajeros son, en la mayoría, CCAA con elevada población o con una mejor situación económica. Por su parte, las CCAA con saldo positivo, y por tanto receptoras netas de viajeros, son el resto de CCAA, destacando, en primer lugar, Andalucía, seguida de Castilla-León, Canarias y Baleares.

La Tabla 2 pone de relieve que Andalucía es la comunidad autónoma con el volumen de gasto promocional más elevado. Centrándonos en el año 2013, a Andalucía le siguen Cataluña, la Comunidad Valenciana y Baleares. No obstante, en el año 2000 este ranking, por volumen de gasto promocional, era distinto, puesto que tras Andalucía aparecía la Comunidad Valenciana, seguida de Canarias y a bastante distancia Galicia. Este hecho pone de relieve el esfuerzo que han realizado algunas CCAA por posicionarse turísticamente entre el año 2000 y 2013. De esta forma, si atendemos a la variación media, a lo largo del periodo considerado, destacan cinco CCAA, que presentan crecimientos medios muy elevados, como son La Rioja, Cataluña, Baleares, Castilla-La Mancha y Castilla-León. Por su parte, Madrid y Canarias son las dos CCAA que experimentan la mayor reducción media en sus gastos promocionales.

Tabla 2

Gastos de promoción de los destinos turísticos por comunidad autónoma

\begin{tabular}{|c|c|c|c|c|c|}
\hline & \multicolumn{2}{|l|}{2000} & \multicolumn{2}{|l|}{2013} & \multirow{2}{*}{$\begin{array}{l}\text { Crecimiento medio } \\
2000-2013\end{array}$} \\
\hline & $\begin{array}{c}\text { Gasto promocional } \\
\text { miles de euros }\end{array}$ & $\%$ & \begin{tabular}{|c|}
$\begin{array}{c}\text { Gasto promocional } \\
\text { miles de euros }\end{array}$ \\
\end{tabular} & $\%$ & \\
\hline Andalucía & 110650.56 & 34.95 & 151491.61 & 34.77 & 1.31 \\
\hline Aragón & 7512.65 & 2.37 & 3897.88 & 0.89 & -4.81 \\
\hline Asturias & 9143.96 & 2.89 & 11124.27 & 2.55 & 1.52 \\
\hline Baleares & 14017.32 & 4.43 & 39305.91 & 9.02 & 8.77 \\
\hline Canarias & 38369.59 & 12.12 & 11491.95 & 2.64 & -8.04 \\
\hline Cantabria & 8368.72 & 2.64 & 7377.51 & 1.69 & -0.27 \\
\hline Castilla y León & 9748.73 & 3.08 & 25429.27 & 5.84 & 7.15 \\
\hline Castilla - La Mancha & 3686.38 & 1.16 & 11453.39 & 2.63 & 8.71 \\
\hline Cataluña & 14994.17 & 4.74 & 49725.41 & 11.41 & 10.16 \\
\hline C. Valenciana & 45514.73 & 14.38 & 39319.01 & 9.03 & -0.43 \\
\hline Extremadura & 2704.91 & 0.85 & 5682.26 & 1.30 & 5.95 \\
\hline Galicia & 18339.24 & 5.79 & 26732.99 & 6.14 & 1.93 \\
\hline Madrid & 5773.35 & 1.82 & 2404.15 & 0.55 & -7.28 \\
\hline Murcia & 15055.08 & 4.76 & 9334.13 & 2.14 & -5.23 \\
\hline Navarra & 2605.23 & 0.82 & 3383.06 & 0.78 & 0.49 \\
\hline País Vasco & 6479.47 & 2.05 & 9284.63 & 2.13 & 1.77 \\
\hline La Rioja & 3639.28 & 1.15 & 28197.10 & 6.47 & 16.84 \\
\hline Total & 316603.35 & 100.00 & 435634.53 & 100.00 & 2.27 \\
\hline
\end{tabular}

Fuente: Elaboración propia a partir de los datos de la Intervención General de la Administración del Estado. 
Por su parte, en la Tabla 3 se pone de relieve que la Comunidad de Madrid, la Comunidad Valenciana, País Vasco y Andalucía son las CCAA que han experimentado un mayor crecimiento medio, en el periodo 2000-2013, en el volumen de viajeros recibidos. No obstante, se puede comprobar, a través de la Figura 1, que la crisis económica supuso un freno al continuo crecimiento que venían experimentando el número de viajeros.

Tabla 3

Viajeros de los destinos turísticos

\begin{tabular}{|l|r|r|r|r|r|}
\hline & \multicolumn{2}{|c|}{$\mathbf{2 0 0 0}$} & \multicolumn{2}{c|}{$\mathbf{2 0 1 3}$} & \multirow{2}{*}{$\begin{array}{c}\text { Crecimiento * } \\
\mathbf{2 0 0 0 - 2 0 1 3}\end{array}$} \\
\cline { 2 - 5 } & Viajeros & \multicolumn{1}{|c|}{$\%$} & Viajeros & $\%$ & 2.89 \\
\hline Andalucía & 5943519 & 18.54 & 8611659 & 20.27 & 0.87 \\
Aragón & 1501469 & 4.68 & 1680966 & 3.96 & 1.99 \\
Asturias & 944164 & 2.94 & 1219651 & 2.87 & 0.13 \\
Baleares & 1108492 & 3.46 & 1128000 & 2.66 & 2.27 \\
Canarias & 1412131 & 4.40 & 1890386 & 4.45 & 0.87 \\
Cantabria & 744495 & 2.32 & 833642 & 1.96 & 0.65 \\
Castilla y León & 2885024 & 9.00 & 3138177 & 7.39 & -0.41 \\
Castilla - La Mancha & 1518491 & 4.74 & 1440086 & 3.39 & 2.11 \\
Cataluña & 4536132 & 14.15 & 5951139 & 14.01 & 3.24 \\
C. Valenciana & 2969353 & 9.26 & 4497032 & 10.59 & 0.29 \\
Extremadura & 952196 & 2.97 & 988241 & 2.33 & 1.57 \\
Galicia & 2078835 & 6.48 & 2545254 & 5.99 & 4.20 \\
Madrid & 3020648 & 9.42 & 5156439 & 12.14 & 2.23 \\
Murcia & 647825 & 2.02 & 863110 & 2.03 & 2.47 \\
Navarra & 449276 & 1.40 & 616973 & 1.45 & 3.03 \\
País Vasco & 1017812 & 3.17 & 1500009 & 3.53 & 1.78 \\
La Rioja & 335481 & 1.05 & 421828 & 0.99 & $\mathbf{1 . 7 8}$ \\
\hline Total & $\mathbf{3 2 0 6 5 3 4 3}$ & $\mathbf{1 0 0 . 0 0}$ & $\mathbf{4 2 4 8 2 5 9 2}$ & $\mathbf{1 0 0 . 0 0}$ & \\
\hline
\end{tabular}

* Es el crecimiento medio para el periodo 2000-2013.

Fuente: Elaboración propia a partir de los datos del INE.

Los datos de la Tabla 3 junto con los de la Tabla 2 permiten apreciar la eficiencia en los gastos de promoción de las CCAA. Se constata que algunas CCAA como Castilla-León, Castilla-La Mancha, Baleares y Extremadura son ineficientes en la utilización de sus gastos de promoción, puesto que incrementan mucho esos gastos, pero no se traduce en un aumento del número de viajeros recibidos, reduciendo su cuota de mercado. Por el contrario, la Comunidad de Madrid y la Comunidad Valenciana son CCAA eficientes en el uso de sus gastos de promoción, puesto que ven incrementados sustancialmente el número de viajeros que reciben, a la vez que reducen la partida de gastos dedicada a promocionar la CCAA, como destino turístico. En ambos casos se observa que han ganado cuota de mercado con respecto al año 2000. 
Figura 1

Gráfico temporal del número de viajeros

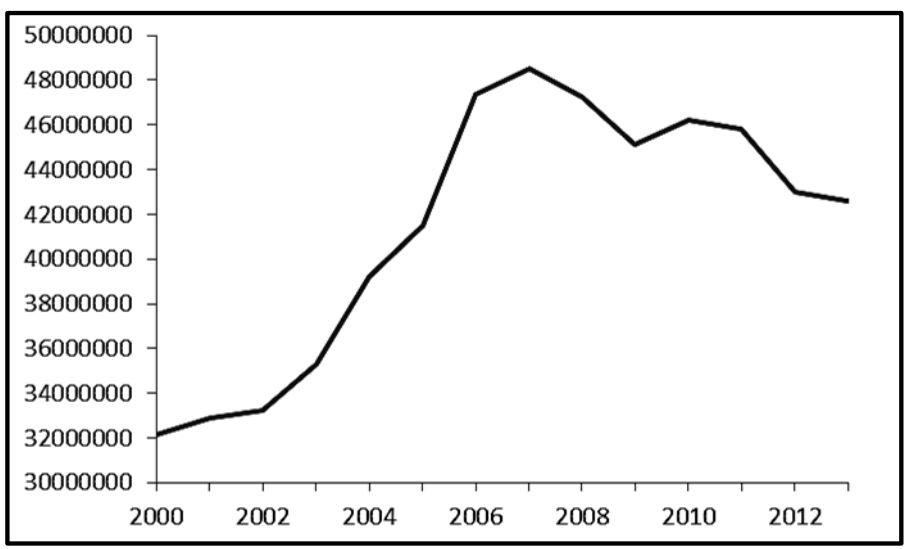

Fuente: INE

Con el fin de estudiar la incidencia de los gastos públicos de promoción sobre el incremento del número de viajeros se ha calculado el coeficiente de correlación entre los gastos de promoción y el incremento de los viajeros obteniendo un coeficiente de correlación estadísticamente significativo e igual a 0.35 , confirmado la hipótesis de partida, de que los gastos promocionales inciden positivamente en el flujo de turistas que se reciben.

En los gráficos de dispersión (ver Figura 2) se constata la correlación positiva entre los gastos de promoción y el número de viajeros que recibe cada CCAA. No obstante, en la represetación gráfica de los datos se observa una mayor dispersión en el año 2000 que en el año 2013. Nuevamente, estos gráficos evidencian la eficiencia de Madrid, que avanza posiciones y se sitúa más cerca del nivel de Andalucía, por lo que respecta al volumen de viajeros, pero con un menor volumen de gasto promocional, y la ineficiencia de algunas de las CCAA como Baleares, que aumenta considerablemente su gasto promocional pero apenas avanza en el volumen de viajeros que recibe.

La Figura 3 permite realizar una primera aproximación para analizar si el efecto del gasto promocional es distinto dentro que fuera de la propia CCAA. Como se puede observar, algunas CCAA cambian su posición, según se considere el crecimiento medio de los viajeros totales o el crecimiento medio de los viajeros procedentes de otras CCAA. Esto pone de relieve que los gastos de promoción, en algunas CCAA, influyen de forma distinta a los turistas locales que a los turistas procedentes de otras CCAA. Entre las CCAA que varían sustancialmente su posición en el gráfico, según se considere el crecimiento medio de los turistas totales o de los turistas procedentes de otras zonas, resaltan: Baleares, Canarias, Murcia, Cataluña y Andalucía. Asimismo, la Figura 2 permite 
también comprobar que Madrid es la Comunidad Autónoma más eficiente de todas, mientras que la Comunidad de Castilla-La Mancha es la más ineficiente. Madrid reduce significativamente su gasto promocional, a la vez que aumenta el volumen de viajeros, mientras que Castilla-La Mancha aumenta su gasto en promoción turística y reduce los viajeros que recibe.

Figura 2

Gráficos de dispersión entre los gastos de promoción del destino y el número de viajeros en los años 2000 y 2013

(en logaritmos neperianos)
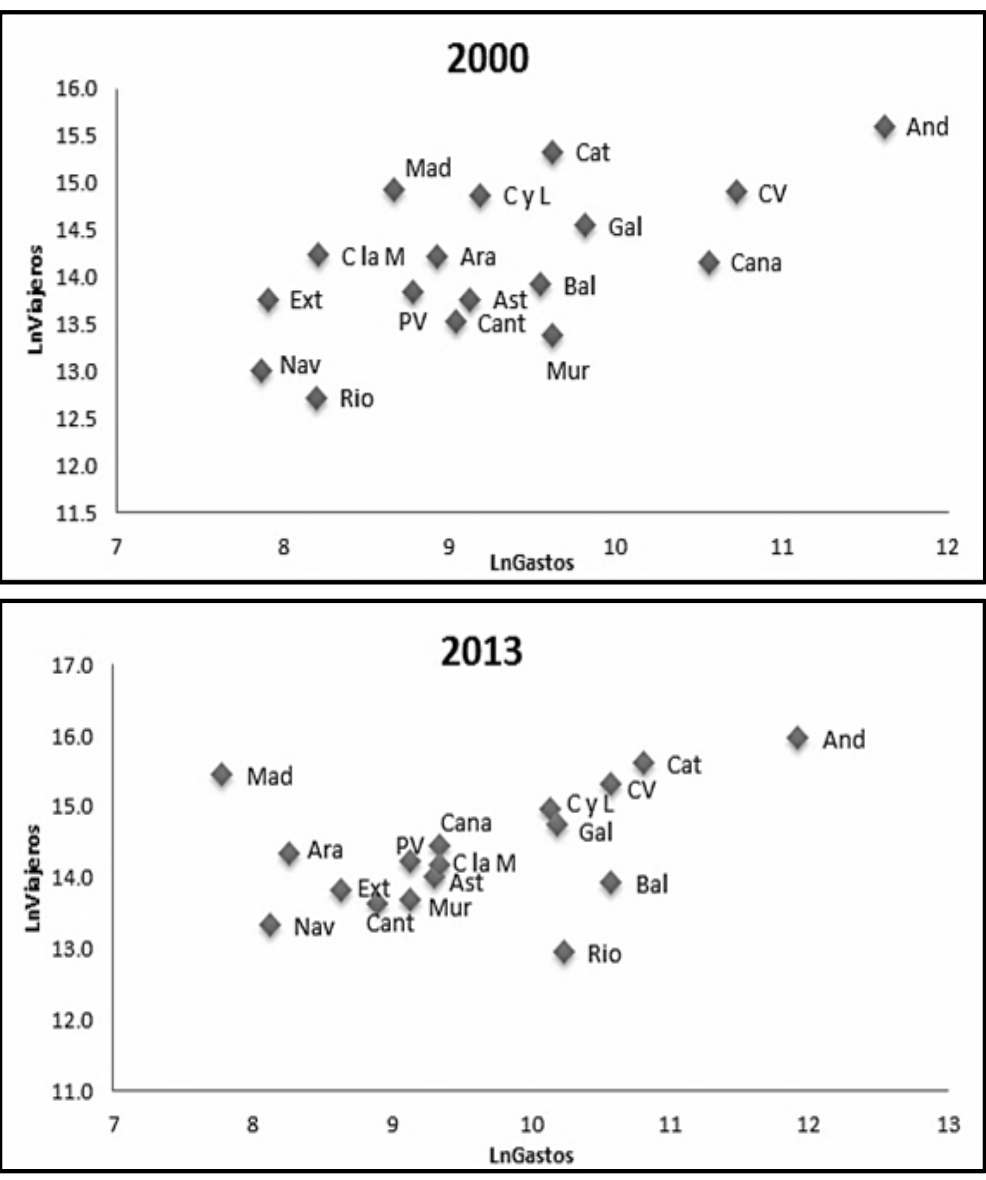

Nota: And=Andalucía, Ara=Aragón, Ast=Asturias, Bal=Baleares, Cana=Canarias, Cant=Cantabria CyL=Castilla y León, C la M=Castilla-La Mancha, Cat=Cataluña, CV=Comunidad Valenciana, Ext=Extremadura, Gal=Galicia, Mad=Madrid, Mur=Murcia, Nav=Navarra, PV=País Vasco, Rio=Rioja.

Fuente: Elaboración propia a partir de los datos del INE. 


\section{Figura 3}

Gráficos de dispersión entre el crecimiento medio del gasto promocional y de los viajeros para el periodo $2000-2013$
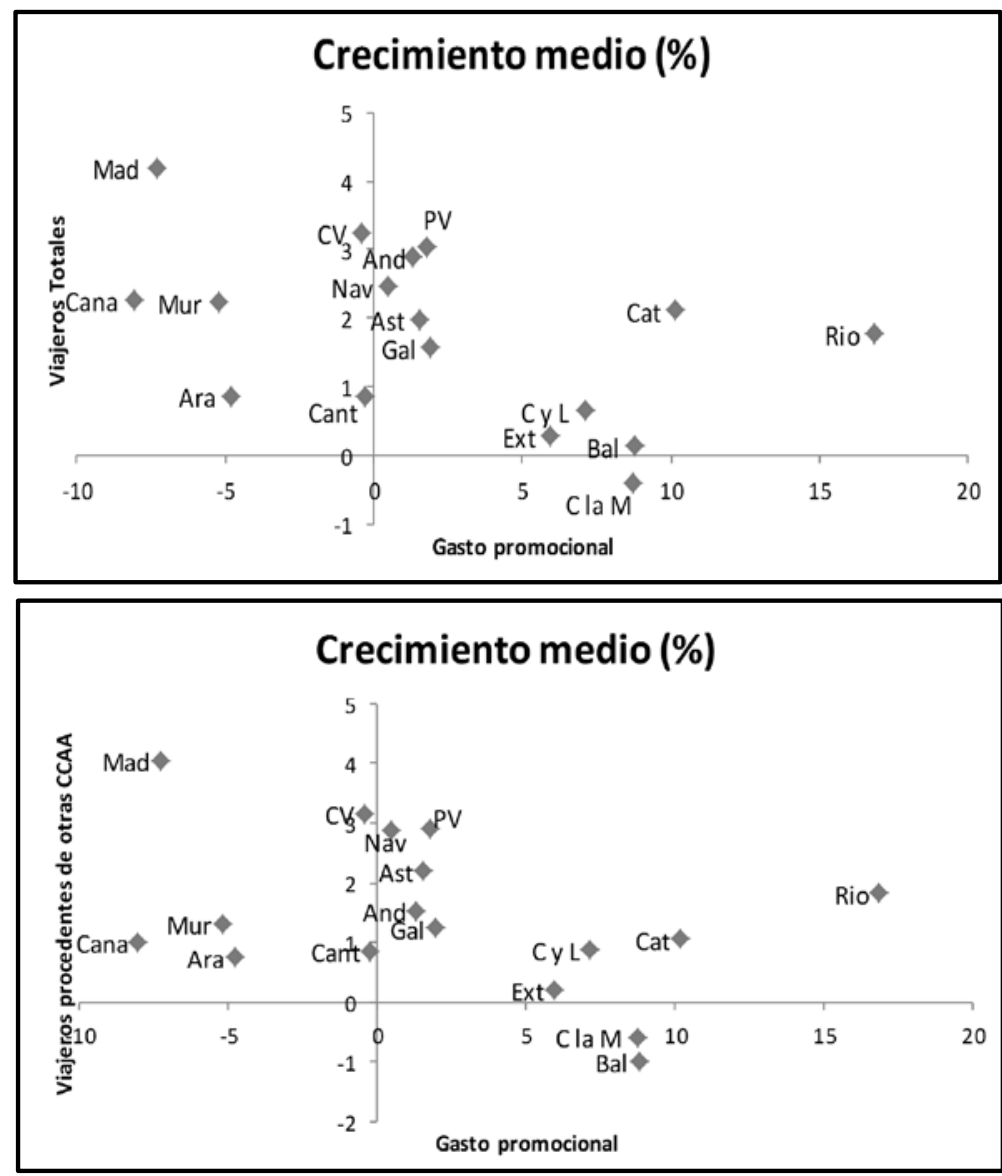

Nota: And=Andalucía, Ara=Aragón, Ast=Asturias, Bal=Baleares, Cana=Canarias, Cant=Cantabria, CyL=Castilla y León, C la $M=$ Castilla-La Mancha, Cat=Cataluña, CV=Comunidad Valenciana, Ext=Extremadura, Gal=Galicia, Mad=Madrid, Mur=Murcia, Nav=Navarra, PV=País Vasco, Rio=Rioja.

Fuente: Elaboración propia a partir de los datos del INE.

Con el objetivo de agrupar las CCAA, según la eficiencia en su gasto promocional, se ha aplicado un análisis clúster, considerando como factores de agrupamiento el crecimiento medio, para el periodo considerado, del gasto promocional, del volumen de viajeros totales y del volumen de viajeros procedentes de otras CCAA. Este análisis de conglomerados ha dado como resultado cuatro clústeres bien diferenciados, pudiéndose distingir entre CCAA muy ineficientes, ineficientes, eficientes y muy eficientes en el aprovechamiento del gasto público de promoción del destino turístico. Las medias de los factores de 
agrupamiento, de cada uno de los clústeres, aparecen recogidas en la Tabla 4. El clúster que incluye a las CCAA más ineficientes son: Baleares, Castilla-La Mancha, Castilla y León y Extremadura. En estas CCAA, los gastos de promoción crecen en más del doble que los gastos medios de todas las CCAA, pero el volumen de viajeros totales apenas varia y los viajeros procedentes de otras CCAA se reducen. Las CCAA ineficientes son: La Rioja y Cataluña. Estas CCAA ven aumentado, por encima de la media, el volumen de turistas que reciben, pero lo hacen aumentando sus gastos de promoción en más de cinco veces el crecimiento medio de todas las CCAA. Las CCAA eficientes en el gasto de promoción son: Andalucía, Asturias, Navarra y Galicia. Estas CCAA aumentan su gasto de promoción pero ven aumentar el volumen de viajeros que reciben, tanto locales como procedentes de otras CCAA, por encima de la media. Finalmente, el clúster que incluye a las CCAA más eficientes está formado por Aragón, Canarias, Cantabria, Comunidad Valenciana, Madrid y Murcia. Estas CCAA reducen sus gastos de promoción, a la vez que aumentan su volumen de viajeros por encima de la media de todas las CCAA.

Tabla 4

Medias de la tasa de variación en porcentaje de los clústeres de las CCAA

\begin{tabular}{|lccccc|}
\hline & Total & $\begin{array}{c}\text { Muy } \\
\text { Ineficientes }\end{array}$ & Ineficientes & Eficientes & $\begin{array}{c}\text { Muy } \\
\text { Eficientes }\end{array}$ \\
\hline Gasto promocional & 2.27 & 7.64 & 13.50 & 1.40 & -4.34 \\
Viajeros totales & 1.78 & 0.17 & 1.94 & 2.39 & 2.28 \\
Viajeros procedentes de otras CCAA & 1.43 & -0.12 & 1.44 & 2.15 & 1.86 \\
\hline
\end{tabular}

Fuente: Elaboración propia.

Otro hecho que se observa en la Tabla 4 es que, en todos los clústeres, el incremento de los viajeros totales es mayor al de los viajeros que proceden de otras CCAA. Esto apunta a que los gastos de promoción han tenido un mayor efecto en los turistas locales que sobre los procedentes de otras zonas.

\section{RESULTADOS}

En la Tabla 5 se recogen los resultados de la estimación del modelo gravitatorio con datos del panel. La utilización de un panel proporciona varias ventajas respecto a la utilización de datos de corte transversal. En primer lugar, proporciona información de la evolución del número de viajeros a lo largo del tiempo. En segundo lugar, permite tener en cuenta los efectos específicos inobservados de cada región, tales como son el clima, la cultura, entre otros, y en tercer lugar, permite capturar las relaciones relevantes entre las variables a lo largo del tiempo.

En la especificación final del modelo estimado, tal y como se apuntó en el apartado de metodología, se han incluido variables ficticias referentes a las re- 
giones de origen y destino, con el fin de recoger los efectos específicos de las CCAA y evitar posibles problemas de errores de especificación.

Tabla 5

Resultados de la estimación del modelo gravitatorio

\begin{tabular}{|c|c|c|c|c|}
\hline & \multicolumn{2}{|c|}{ MCO } & \multicolumn{2}{|c|}{ VI } \\
\hline & Coeficiente & Desv. Estándar & Coeficiente & Desv. Estándar \\
\hline $\operatorname{Ln}\left(F_{i j t-1}\right)$ & $0.174^{\star \star \star}$ & $(0.013)$ & $0.126^{\star \star \star}$ & $(0.023)$ \\
\hline $\ln \left(\mathrm{GP}_{\mathrm{it}}\right)$ & $0.121^{\star \star \star}$ & $(0.016)$ & $0.111^{* * *}$ & $(0.017)$ \\
\hline $\ln \left(\mathrm{PIB}_{\mathrm{it}}\right)$ & -0.043 & $(0.054)$ & -0.039 & (0.054) \\
\hline $\ln \left(P \mid B_{j t}\right)$ & $0.516^{\star \star \star}$ & (0.108) & $0.546^{\star \star \star}$ & $(0.111)$ \\
\hline $\ln \left(\mathrm{PH}_{\mathrm{it}}\right)$ & $-2.133^{\star \star \star}$ & $(0.186)$ & $-2.194^{\star \star \star}$ & (0.203) \\
\hline $\ln \left(N P_{i t}\right)$ & $0.469 * \star \star$ & $(0.021)$ & $0.493^{\star * \star}$ & $(0.020)$ \\
\hline $\mathrm{S}_{\mathrm{ij}}$ & $0.364^{\star \star *}$ & $(0.175)$ & $0.506^{\star \star \star}$ & $(0.181)$ \\
\hline $\ln \left(D_{\mathrm{ij}}\right)$ & $-0.278^{\star \star \star}$ & $(0.027)$ & $-0.256^{\star \star \star}$ & $(0.027)$ \\
\hline Cont $_{\mathrm{ij}}$ & $0.554^{\star \star * *}$ & $(0.030)$ & $0.581^{\star \star *}$ & $(0.032)$ \\
\hline$|s|_{j}$ & $-0.086^{\star \star \star}$ & $(0.006)$ & $-0.094^{\star \star \star}$ & $(0.007)$ \\
\hline Isla & $0.014^{\star \star \star}$ & $(0.005)$ & $0.011^{\star \star}$ & $(0.006)$ \\
\hline $\mathrm{CM}_{\mathrm{j}}$ & $0.029 * \star \star$ & $(0.006)$ & $0.022^{\star \star \star}$ & (0.006) \\
\hline $\mathrm{CM}_{\mathrm{i}}$ & $0.151^{\star \star \star}$ & $(0.006)$ & $0.156^{\star \star \star}$ & $(0.007)$ \\
\hline $\mathrm{CA}_{\mathrm{j}}$ & $0.030^{\star \star \star}$ & (0.003) & $0.025^{\star \star \star}$ & (0.003) \\
\hline $\mathrm{CA}_{\mathrm{i}}$ & $0.087^{\star \star \star}$ & $(0.004)$ & $0.090^{\star \star \star}$ & (0.005) \\
\hline $\operatorname{Mad}_{\mathrm{i}}$ & $0.124^{\star \star \star}$ & $(0.007)$ & $0.119^{\star \star \star}$ & $(0.007)$ \\
\hline $\operatorname{Mad}_{j}$ & $0.202^{* \star *}$ & (0.013) & $0.210^{\star \star \star}$ & (0.014) \\
\hline Dummies $C_{C A A_{j}}$ & $\mathrm{Si}$ & & $\mathrm{Si}$ & \\
\hline Dummies $C C A A_{i}$ & Si & & Si & \\
\hline Dummies temporal & No & & No & \\
\hline Constante & $11.918^{\star \star \star}$ & $(0.918)$ & $12.344^{\star * *}$ & $(1.044)$ \\
\hline Número de observaciones & 3756 & & 3466 & \\
\hline $\mathrm{R}^{2}$ Corregido & 0.830 & & 0.845 & \\
\hline AIC & -1.151 & & -1.242 & \\
\hline Contraste Endogeneidad (1) & 43.919 & & - & \\
\hline ( $p$-value) & $(0.000)$ & & & \\
\hline
\end{tabular}

Nota: ** Significatividad al 1\%, ** Significaividad al $5 \%$.

MCO= Mínimos cuadrados ordinarios robustos a la presencia de autocorrelación y hetorocedasticidad. VI= Método de Variables Instrumentales robusto a la presencia de autocorrelación y heterocedasticidad.

(1) Test de Durbin, Wu y Hausman.

Fuente: Elaboración propia.

El modelo se ha estimado, inicialmente, por MCO, robustos a la posible presencia de autocorrelación y heterocedasticidad, y se ha contrastado la endogeneidad en el modelo, mediante el test de Durbin, Wu y Hausman (Nakamura y Nakamura, 1981) ${ }^{9}$. El rechazo de la hipótesis de exogeneidad obliga a utilizar

\footnotetext{
${ }^{9}$ El estudio de la endogeneidad se ha centrado en el regresando desfasado, utilizando el estadístico de Durbin, Wu y Hausman. No obstante, se ha estudiado la posible endogenidad de otros regresores pero, en todos los casos, se ha llegado a la conclusión de que su comportamiento era exógeno.
} 
un método de estimación consistente, como es el método de variables instrumentales robusto $(\mathrm{VI})^{10}$.

Centrándonos en la estimación del modelo por VI, que resulta ser el adecuado, se aprecia que la demanda turística interior presenta inercia y, por lo tanto, destaca la existencia de un efecto dinámico de fidelización del destino turístico y de atracción por la experiencia. En concreto el coeficiente estimado indica que en promedio el $12.6 \%$ de los flujos turísticos en t para un destino turístico vienen determinados por los flujos en el periodo anterior. Este resultado es similar a los obtenidos en otros trabajos, como Garín-Muñoz (2009) y Khadaroo y Seetanah (2008). Al calcular el valor del retardo medio, éste se sitúa en 0.14 , pudiéndose concluir que la demanda turística interna se ajusta en un periodo de tiempo relativamente corto, adaptándose a las exigencias de los clientes.

Por lo que respecta a la variable gastos de promoción del destino turístico, su coeficiente estimado indica que un incremento del $1 \%$ de los gastos de promoción aumenta el flujo de viajeros en un $0.11 \%$ en el corto plazo y un $0.13 \%$ en el largo plazo. Este efecto positivo y significativo de los gastos de promoción del destino turístico, realizados por las autoridades para atraer a los turistas, justifican la importancia que tienen las políticas públicas encaminadas a la promoción de los destinos turísticos.

El PIB per cápita de la zona de origen afecta positivamente al flujo de turistas ya que es un indicador de la capacidad de compra de los potenciales turistas locales. La elasticidad del PIB de origen es un indicador de que el gasto turístico no es un artículo de lujo. Por su parte, el PIB per cápita de la zona de destino no resulta ser estadísticamente significativo.

Los precios de los servicios hoteleros son factores estadísticamente significativos a la hora de explicar los viajes interregionales. En concreto, un incremento del $1 \%$ de esta variable reduce la demanda hotelera de la región en un $2.2 \%$ en el corto plazo y en un $2.5 \%$ en el largo plazo. Estos resultados ponen de relieve lo sensible que es la demanda turística a variaciones en los precios hoteleros y la importancia que tiene en España las funciones de coste de los empresarios del sector.

El número de plazas de la zona de destino ejerce un efecto atracción positivo y significativo, dado que es un indicador de la oferta en el destino turístico y del poder de elección de oferta para el cliente.

La distancia, efectivamente, ejerce un efecto fricción, como señala Reilly (1931), presentando signo negativo y siendo altamente significativa, indicando

${ }^{10}$ Como instrumentos se han utilizado todas las variables que se han considerado exógenas, junto con sus respectivos lags. Además también se ha considerado como variable instrumental la variable endógena con dos desfases. 
que aquellos destinos más distantes tendrán un menor flujo de turistas entre sí, ya que tendrán un coste más elevado, como era de esperar.

La variable que capta el efecto frontera verifica la existencia de un fuerte efecto. En concreto, el flujo de viajeros procedentes de la misma CCAA es 1.7 veces mayor al de los viajeros procedentes de otras CCAA. Por otro lado, la contigüidad de las CCAA también afecta positivamente al flujo de turistas. Por lo que respecta a la insularidad, cuando las islas son el origen el signo del coeficiente es negativo, pero cuando son el destino es positivo, recogiendo el atractivo turístico específico que éstas presentan. Las CCAA con costa, tanto mediterránea como atlántica, presentan un signo positivo y un mayor valor cuando son CCAA de destino que cuando son de origen.

En el caso de la condición de capitalidad del Estado, tanto cuando es el origen como cuando es el destino, se obtiene un signo positivo del coeficiente, puesto que la condición de capitalidad hace que existan flujos de viajeros de y hacia la capital del Estado, por motivos de trabajo o de estudios. No obstante, el valor del coeficiente es mucho mayor cuando es el destino, que cuando es el origen de los flujos.

En definitiva el modelo de gravedad estimado corrobora las expectativas depositadas en él, ya que los parámetros estimados, en su mayoría, son altamente significativos, todos presentan el signo esperado y la bondad de ajuste es elevada. Además, se comprueba la relevancia de la variable gastos de promoción que se puede considerar como una variable estratégica para conseguir medidas de política turística activas.

El estudio de los efectos diferenciales, atendiendo a las zonas geográficas de destino, permite distinguir la eficacia y rentabilidad de los gastos públicos de promoción. A tal fin se ha introducido en el modelo econométrico, ecuación (4) y modelo estimado mediante VI de la Tabla 5, una variable que recoge la interacción del gasto promocional con las variables que recogen las distintas zonas de destino del turismo interior. Se han analizado las zonas de destino siguientes: a) cuando la comunidad de origen es contigua de la de destino b) cuando la comunidad de origen y la destino es la misma c) cuando se considera la distancia kilométrica entre la comunidad de origen y la de destino.

Los resultados de las estimaciones de los efectos diferenciales se presentan en la Tabla 6. Dicha tabla tan solo incluye los resultados de las variables relevantes relacionadas con el gasto público de promoción turística. El Modelo 1 es el mismo que el modelo estimado mediante VI de la Tabla 5. En dicho modelo se pone de manifiesto que en general la elasticidad a corto plazo del número de viajeros respecto a los gastos de promoción es del 0.111. Apoyándonos en el Modelo 2 se desprende que para el colectivo formado por los viajeros de las CCAA vecinas (contiguas) el poder de atracción de los gastos públicos de promoción no es significativo. 
Tabla 6

Análisis de sensibilidad de los gastos de promoción

\begin{tabular}{|c|c|c|c|c|}
\hline & Modelo 1 & Modelo 2 & Modelo 3 & Modelo 4 \\
\hline $\operatorname{Ln}\left(\mathrm{GP}_{\mathrm{it}}\right)$ & $\begin{array}{c}0.111^{\star * \star} \\
(0.017)\end{array}$ & $\begin{array}{c}0.109^{\star \star \star} \\
(0.019)\end{array}$ & $\begin{array}{c}0.093^{\star \star \star} \\
(0.016)\end{array}$ & $\begin{array}{c}0.282^{\star * *} \\
(0.056)\end{array}$ \\
\hline $\operatorname{Ln}\left(\mathrm{GP}_{\mathrm{it}}\right)^{\star} \operatorname{Cont}_{\mathrm{ij}}$ & - & $\begin{array}{c}0.009 \\
(0.021)\end{array}$ & - & - \\
\hline $\operatorname{Ln}\left(G_{P}\right)^{*} S_{i j}$ & - & - & $\begin{array}{l}0.241^{\star * \star} \\
(0.060)\end{array}$ & - \\
\hline $\operatorname{Ln}\left(G_{P_{i t}}\right)^{\star} \operatorname{Ln}\left(D_{i j}\right)$ & - & - & - & $\begin{array}{c}-0.030 \star \star \star \\
(0.009)\end{array}$ \\
\hline Número de observaciones & 3466 & 3466 & 3466 & 3466 \\
\hline Número de variables & 26 & 27 & 27 & 27 \\
\hline AIC & -1.248 & -1.248 & -1.256 & -1.253 \\
\hline
\end{tabular}

A partir de los resultados del Modelo 3, de la Tabla 6, se pone de manifiesto que los flujos turísticos dentro de una misma comunidad autónoma presentan una elasticidad diferencial a corto plazo de 0.241 , de tal forma que la elasticidad total es de 0.334 . De ello se deduce que los gastos de promoción turística de una comunidad poseen un gran poder disuasorio a desplazarse a otras CCAA, de forma que sus ciudadanos realizan su actividad turística dentro de sus propios límites.

En cuanto a la importancia del efecto combinado de la distancia kilométrica con los gastos de promoción del destino turístico, ver Modelo 4, se concluye que la variable resultante es estadísticamente significativa, pero su incidencia sobre el flujo de los viajeros es muy reducida. En efecto, dado que el valor del coeficiente (-0.030) es exiguo se pone de manifiesto el hecho de que la distancia kilométrica no supone una barrera para que los viajeros realicen un desplazamiento desde las comunidades más alejadas. En definitiva los gastos de promoción de una comunidad autónoma ejercen un poder de atracción sobre los flujos turísticos interiores, donde la distancia kilométrica no supone una restricción elevada a la recepción de viajeros.

\section{CONCLUSIONES}

El análisis de los flujos turísticos a través de las matrices origen-destino es una herramienta eficaz para entender el comportamiento de la demanda turística interior de España y de los factores que inciden sobre ella. Sin embargo, en los trabajos existentes hasta la fecha, no se había considerado como variable explicativa el gasto público de promoción de los destinos turísticos. En este trabajo se ha puesto de manifiesto su elevada relevancia como indicador de la demanda 
interior y el poder explicativo de los gastos de promoción. Asimismo, este trabajo utiliza un modelo de gravedad dinámico que implica utilizar un método de estimación adecuado, como es el método de variables instrumentales.

La aportación de este artículo, entre otras, es el tratamiento específico de la variable promoción. Dicha variable influye significativamente sobre la atracción de turistas confirmando la hipótesis de partida de que los gastos de promoción influyen positivamente en los flujos turísticos internos y posicionan a las distintas CCAA. Igualmente se ha apreciado que los gastos de promoción del destino no son igualmente eficientes en todas las comunidades. Para ello se ha realizado un análisis clúster que ha permitido concluir que las comunidades más eficientes, en la promoción turística, son Aragón, Canarias, Cantabria, Comunidad Valenciana, Madrid y Murcia.

El análisis de los datos ha puesto de relieve que la crisis económica pasó factura a la demanda turística interior y se ha analizado de qué manera la contigüidad, la distancia y las características económicas inciden en los flujos turísticos interiores. En concreto, la mejora de la capacidad de compra de las zonas de origen, con la recuperación económica, ha impulsado la demanda turística, tras el freno que experimentó con la crisis. Por otro lado, la distancia actúa como un elemento de fricción, reduciendo los flujos turísticos entre regiones alejadas mientras que los gastos de promoción influyen positivamente en la demanda turística.

Por tanto, de este trabajo se obtienen conclusiones interesantes sobre la importancia, para la fijación de la demanda turística, del PIB per cápita de la zona de origen. Dicho resultado es totalmente coherente con la literatura al uso ya que, de forma aproximada, lo que cuantifica esta variable es la capacidad de compra de los potenciales viajeros. Asimismo, se ha comprobado que el término inercia es significativo, recogiendo el efecto fidelización del destino por parte del turista.

En definitiva el modelo de gravedad estimado corrobora las expectativas depositadas en él, ya que los parámetros estimados, en su mayoría, son altamente significativos, todos presentan el signo esperado y su bondad de ajuste es elevada.

Por último, el estudio de los efectos diferenciales ha permitido cuantificar los efectos de los gastos de promoción sobre los flujos turísticos de distintos colectivos de viajeros de España. Los resultados obtenidos, con especial atención a las distintas zonas de destino, permiten concluir que el máximo poder de atracción realizado por los gastos de promoción de un destino turístico, se ejerce sobre los viajeros cuyo origen es la propia comunidad autónoma, marcando así una diferencia significativa entre comunidades especializadas en la emisión turismo doméstico y las receptoras de turismo interior. 


\section{REFERENCIAS BIBLIOGRÁFICAS}

AKAL, M. (2004). "Forecasting Turkey's tourism revenues by ARMAX model". Tourism Management, 25, pp. 565-580.

ALEGRE, J. y POU, L. (2004). "La participación de las familias españolas en el consumo turístico". Papeles de Economía Española, 102, pp. 107-24.

ALMAGRO, J. (1979). "Aplicaciones del enfoque Box-Jenkins a series del turismo español". Cuadernos Económicos del ICE, 11-12, pp. 55-107

ANDERSON, J.E. y VAN WINCOOP, E. (2003). "Gravity with Gravitas: A Solution to the Border Puzzle". American Economic Review, 93(1), pp. 170-192.

ANDERSON, J.E. y VAN WINCOOP, E. (2004). "Trade Costs"'. Journal Economic Literature, 42(3), pp. 691-751.

ANDERSON, J.E. (2011). "The Gravity Model". Annals Review of Economics, 3(1), pp. 133-160.

ATHANASOPOULOS, G. y HYNDMAN, R.J. (2008). "Modelling and forecasting Australian domestic tourism". Tourism Management, 29(1), pp. 19-31.

AZNAR, J. y NICOLINI, R. (2007). "El sector turístico en la Comunidad Valenciana: Unos elementos de análisis de la demanda en el marco de la economía geográfica". Revista de Estudios Regionales, 79, pp. 43-72.

BERTOLI, S. y FERNÁNDEZ-HUERTAS, J. (2013). "Multilateral resistance to migration". Journal of Development Economics, 102, pp. 79-100

BOX, G.E.P. y JENKINS, G. (1970). Time Series Analysis, Forecasting and Control. San Francisco: Holden-Day.

CABRER, B. e IRANZO, D. (2007). "El efecto de los atentados del 11-S sobre el turismo en España". Revista de Estudios de Economía Aplicada, 25(1), pp. 365-386.

CAÑADA, A. (2002). "El turismo interior en España y su dimensión espacial: las regiones como exportadoras e importadoras de turismo interior". Cuadernos de Información Económica, 169, pp. 137-140.

CAÑADA, A. (2007). "Cuenta satélite del turismo interior: Una visión macroeconómica de la relevancia del turismo". Revista de Estadística y Sociedad, 24, pp. 6-8.

CHAN, F., LIM, C. y McALEER, M. (2005). "Modelling multivariate international tourism demand and volatility". Tourism Management, 26, pp. 459-471.

CHU, F.L. (2008). "Forecasting tourism demand with ARMA-based methods". Tourism Management, 10, pp. 1-12.

COSHALL, J.T. (2009). "Combining volatility and smoothing forecast of UK demand for international tourism". Tourism Management, 30, pp. 495-511.

CROUCH, G.I. (1995). "A meta-analysis of tourism demand". Annals of Tourism Research, 22, pp. 103-118.

CROUCH, G.I., SCHULTZ, L. y VALERIO, P. (1992). "Marketing international tourism to Australia: A regression analysis". Tourism Management, 13(2), pp. 196-208.

DE LA MATA, T. y LLANO, C. (2010). "Modelo gravitatorio y turismo: Una aplicación a los flujos monetarios interregionales del sector Turismo en España". Revista de Estudios Regionales, 89, pp. 211-240. 
DE LA MATA, T. y LLANO, C. (2012). "Spatial pattern and domestic tourism: An econometric analysis using inter-regional monetary flows by type of journey". Papers in Regional Science, 91(2), pp. 437-470.

ERIKSEN, L. y AHMT, T. (1999). "Measuring and Modeling the Regional. Impact of Tourism in Denmark". International Journal of Tourism Research, 1, pp. 313-327.

FUJI, E.T. y MAK, J. (1981). "Forecasting tourism demand: some methodological issues". Annals of Regional Science, 15, pp. 72-82

GARÍN-MUÑOZ, T. (2008). "Cambios en las tendencias del turismo internacional y sus implicaciones para la economía española". Papeles de Economía Española, 116, 'pp. 79-93.

GARÍN-MUÑOZ, T. (2009). "Tourism in Galicia: domestic and foreign demand". Tourism Economics, 15(4), pp. 753-769.

GARÍN-MUÑOZ, T. (2011). "La demanda de turismo británico en España". Boletín Económico de ICE, 3010, pp. 49-62.

GIL-PAREJA, S., LLORCA, R., MARTINEZ, J.A. y OLIVER, J. (2005). "The Border Effect in Spain". The World Economy, 28, pp. 1617-1631.

$\mathrm{GOH}$, C. y LAW, R. (2002). "Modeling and forecasting tourism demand for arrivals with stochastic nonstationary seasonality and intervention". Tourism Management, 23, pp. 499-510.

GUARDIA, T., MURO, J. y SUCH, M. (2014). "Measuring and analysing domestic tourism: the importance of an origin and destination matrix". Tourism Economics, 20(3), pp. 451-472.

HATANAKA, M. (2015). "Los cambios en el volumen y los flujos en los mercados turísticos a escala internacional”. En Aguiló E. y Antón, S. (coords.): 20 retos para el turismo en España (pp. 27-43). Madrid: Editorial Pirámide.

IMM NG, S., ANNE LEE, J. y SOUTAR, G. N. (2007). "Tourists' intention to visit a country: The impact of cultural distance". Tourism Management, 28, pp. 1497-1506.

JUANEDA, C.N. y RIERA, A. (2011). "La oportunidad de la investigación en economía del turismo". Estudios de Economía Aplicada, 29(3), pp. 711-722.

KHADAROO, J. y SEETANAH, B. (2008). "The role of transport infrastructure in international tourism development: A gravity model approach". Tourism Management, 29, pp. 831-840.

KIM, S.I. y FESENMAIER, D.R. (1990). "Evaluating Spatial Structure Effects in Recreation Travel". Leisure Sciences, 12, pp. 367-381.

LARGO, J. (1976). "El turismo y el mercado interior en España". Estudios Turísticos, 4950, pp. 71-99.

LEE, C.K., SONG, H.J. y MJELDE, J.W. (2008). "The forecasting of international expo tourism using quantitative techniques". Tourism Management, 29, pp. 1084-1098.

LIM, C., (1997). "Review of International Tourism Demand Models". Annals of Tourism Research, 24, pp. 835-849.

LIM, C. (2006). "A survey tourism demand modeling practice: issues and implications". In Edwar Elgar: International Handbook on the Economics of Tourism (pp. 45-72). Cheltenham UK. Northampton. MA, USA.

MALAMUD, B. (1973). "Gravity model calibration of tourist travel to Las Vegas". Journal of Leisure Research, 5(4), pp. 23-33. 
MARTIN, C.A. y WITT, S.F. (1987). "Tourism demand forecasting models: choice of appropriate variable to represent tourists' cost of living". Tourism Management, 8, pp. 233-245.

MASSIDA, C. y ETZO, I. (2012). "The determinants of Italian domestic tourism: A panel data analysis". Tourism Management, 33, pp. 603-610.

McCALLUM, J. (1995). "National borders matter: Canadian-U.S. Regional trade patterns". American Economic Review, 85(3), pp. 615-623.

MILLÁN, M. (2004). "Turismo en la Región de Murcia: evolución de la oferta turística de interior y su distribución espacial”. Cuadernos de Turismo, 13, pp. 51-72.

MORLEY, C.L. (1998). "A dynamic international demand model". Annals of Tourism Research, 25(1), pp. 70-84.

MORLEY, C., ROSSELLÓ, J. y SANTANA-GALLEGO, M. (2014). "Gravity models for tourism demand: theory and use". Annals of Tourism Research, 48, pp. 1-10.

NAKAMURA, A. y NAKAMURA, M. (1981). "On the relationships among several specification error tests presented by Durbin, Wu and Hausman". Econométrica, 49(6), pp. 1583-1588.

NICOLAU, J.L. (2008). "Characterizing Tourist Sensitivity to Distance". Journal of Travel Research, 47, pp. 43-52

OKUBO, T. (2004). "The Border Effect in the Japanese Market: A Gravity Model Analysis". The Japanese and International Economies, 18, pp. 111.

OTERO, J. (1996). "Principales determinantes del flujo de pasajeros extranjeros desembarcados en el aeropuerto de Málaga". Revista de Estudios de Economía Aplicada, 12, pp. 129-142.

PATUELLI, R., MUSSONI, M. y CANDELA, G., (2014). "Cultural offer and distance in spatial interaction model for tourism". WP 14_14, The Rimini Centre For Economic Analysis.

PREEZ, J. y WITT, S.F. (2003). "Univariate versus multivariate time series forecasting: an application to international tourism demand". International Journal of Forecasting, 19, pp. 435-451.

PRIEGO, F.J., ROSSELlÓ, J. y SANTANA-GALLEGO, M. (2015). "The impact of climate change on domestic tourism: a gravity model for Spain". Regional Environment Change, 15, pp. 291-300.

REQUENA F. y LLANO, C. (2010). "The Border Effects in Spain: An Industry-Level Analysis". Empirica. DOI: 10.1007/s10663-010-9123-6.

REILLY, W.J. (1931). The law of retail gravitation. New York, W.J.: Reilly, Inc.

ROSSELLÓ, J. y SANTANA-GALLEGO, M. (2014). "Recent trends in international tourist climate preferences: a revised pictures for climatic change scenarios". Climatic Change, 124, pp. 119-132.

SHELDON, P.J. y VAR, T. (1985). "Tourism Forecasting: A Review of Empirical Research". Journal of Forecasting, 4, pp. 183-195.

SONG, H. y LI, G. (2008). "Tourism demand modeling and forecasting: A review of recent research". Tourism Management, 29, pp. 203-220.

USACH, J. (1998). "Análisis de los flujos interregionales de la demanda turística interna española”. Estudios Turísticos, 136, pp. 27-44.

WITT, S. F. y MARTIN, C. A. (1987). "International tourism demand models inclusion of marketing variables". Tourism Management, 8(1), pp. 33-40. 
WITT, S. F. y WITT, C. A. (1995). "Forecasting tourism demand: A review of empirical research". International Journal of Forecasting, 11, pp. 447-475.

WONG, K., SONG, H., WITT, S.F. y WU, D.C. (2007). "Tourism forecasting: to combine or not combine?". Tourism Management, 28, pp. 1068-1078.

ZHANG, J. y JENSEN, C. (2007). "Comparative Advantage: Explaining Tourism Flows". Annals of Tourism Research, 34, pp. 223-243. 
